\title{
The Effects of Initial Crystalline Morphology on the Properties of Drawn Polypropylene
}

\author{
Masayoshi ITO and Koji TANAKA \\ Department of Chemistry, Science University of Tokyo, \\ Kagurazaka 1-3, Shinjuku-ku, Tokyo 162, Japan
}

(Received January 16, 1986)

\begin{abstract}
Proton spin-spin relaxation times, linear thermal expansivity and tensile modulus have been measured for drawn polypropylene prepared from both bulk-crystallized spherulitic materials (MGC) and solution grown crystals (SGC). Effects of initial morphology are found for chain mobility in the noncrystalline regions and for crystalline orientation function, which produced the difference in the efficiency of draw between MGC and SGC. The mass fraction of the highly strained noncrystalline component $\left(F_{i}\right)$ changed with draw ratio. At higher draw ratio, the stepwise decreases of the MGC were accompanied by corresponding increases in the crystalline mass fraction $\left(F_{\mathrm{c}}\right)$. However, the $F_{\mathrm{c}}$ of the SGC remained almost constant for the draw ratio investigated. A large rubber-elastic contraction observed for highly drawn samples suggests that the axial length of the crystalline bridges is not enough and/or the bridge content is not sufficiently large to resist the contraction. At any draw ratio, the tensile modulus was higher for MGC than for SGC, which indicates the higher efficiency of draw for MGC than for SGC.

KEY WORDS Polypropylene / Crystalline Morphology / Spherulites /

Solution Grown Crystals / Pulsed NMR / Linear Thermal Expansivity /

Mechanical Properties / Efficiency of Draw /
\end{abstract}

Ultradrawing of some semicrystalline polymers produces unusually high tensile modulus and strength. For drawn samples, the important role of the noncrystalline regions which connect two or more crystallites along the fiber direction has been emphasized to explain the improved mechanical properties. ${ }^{1,2}$ However, less information has been obtained on the structure and property changes in the noncrystalline regions as compared with crystalline regions in the drawn materials. In our previous pulsed NMR studies of drawn polyethylene ${ }^{3}$ and polypropylene, ${ }^{4}$ two or three spin-spin relaxation times, corresponding to molecular segments with different mobilities have been observed, depending on draw ratio and draw temperature. The relaxation time for the noncrystalline regions changes drastically with draw ratio, indicating a high sensitivity of the relaxation time to structural changes in the noncrystalline regions.

Ultradrawing of isotactic polypropylene has been achieved by Cansfield et al, ${ }^{5}$ Taylor and Clark, ${ }^{6}$ and Kamezawa et al. ${ }^{7}$ by different techniques. Birefringence, ${ }^{8}$ thermal expansion, ${ }^{9}$ ultrasonic, ${ }^{10,11}$ and dynamic mechani$\mathrm{cal}^{7,10}$ methods have been used to elucidate the structure and properties of drawn polypropylene. However, these works were limited to the samples drawn from bulk-crystallized spherulitic materials. It is widely accepted that the number of inter and intra lamellar tie molecules is larger in bulk-crystallized materials than in solution grown crystals. Such tie molecules are expected to have an important role in the deformation and properties of the resultant drawn materials.

In the present study, the effects of initial crystalline morphology of polypropylene on 
the efficiency of draw were evaluated from the spin-spin relaxation time, linear thermal expansivity and mechanical properties by using the drawn samples prepared from both bulkcrystallized films and solution grown crystal mats. It was found that the initial morphology had a remarkable effect on the properties of drawn materials. Bulk-crystallized sample showed a high efficiency of draw than solution grown crystals.

\section{EXPERIMENTAL}

Isotactic polypropylene(PP) used was kindly supplied by Toa Nenryo Kogyo Co. The viscosity average molecular weight was $\sim 2 \times$ $10^{5}$. The solution grown crystals (SGC) of PP were isothermally precipitated from dilute solutions $(\sim 0.1 \mathrm{wt} \%)$ in xylene kept at $40^{\circ} \mathrm{C}$ for $20 \mathrm{~h}$. Sedimented SGC mats were obtained by slowly filtering the crystal suspension followed by drying in vacuo at room temperature for 2 days. To obtain bulk-crystallized (MGC) films, pellets of PP were compression-molded at $180^{\circ} \mathrm{C}$ into isotropic sheets, which were then cooled at a rate of about $10 \mathrm{~K} \mathrm{~min}^{-1}$ to room temperature. Drawn PP samples were prepared by the coextrusion method. ${ }^{12}$ SGC mats or MGC films were placed between two split bullet halves of linear polyethylene and the assembly co-extruded at $130^{\circ} \mathrm{C}$. The draw ratio was determined by measuring the separation between lateral ink marks on the sample before and after the extrusion. The maximum attainable draw ratio of the SGC and MGC was 23 and 16, respectively. To obtain highly drawn samples of MGC, isotropic MGC films were drawn by tensile tester at $130^{\circ} \mathrm{C}$, which produced the sample with the high maximum draw ratio of 23 .

Pulsed NMR measurements were performed with a JEOL pulsed NMR spectrometer (JSE5B) operating at $60 \mathrm{MHz}$. The proton spinspin relaxation time was obtained by solid echo sequence. Details of NMR measurements and the component resolution of the signal decay are described elsewhere. ${ }^{4}$ The linear thermal expansivity along the extrusion direction $\left(\beta_{\|}\right)$was measured between -100 and $100^{\circ} \mathrm{C}$ at a heating rate of $5 \mathrm{~K} \mathrm{~min}^{-1}$ with a SEIKO DENSHI thermomechanical analyzer (TMA-10). The modulus of the drawn sample along the fiber axis was measured at room temperature on a Tensilon tensile tester (HTM-100). The modulus was determined at a strain rate of $\sim 4 \times 10^{-3} \mathrm{~s}^{-1}$ from the tangent to the stress-strain curve at $0.1 \%$ strain.

\section{RESULTS AND DISCUSSION}

Spin-Spin Relaxation Time and Mass Fraction of the Component

Above room temperature all the samples showed three different relaxation times. Figure 1 shows the three relaxation times as a function of temperature for drawn samples prepared from solution grown crystal mats (SGC series). Only the data for three draw ratios $(\mathrm{DR}=1,10,17)$ are presented. The longest relaxation time $T_{2 \mathrm{a}}$, which is derives from an exponential decay, is ascribed to relaxation of molecules in the non-crystalline regions that are motionally activated on the NMR time

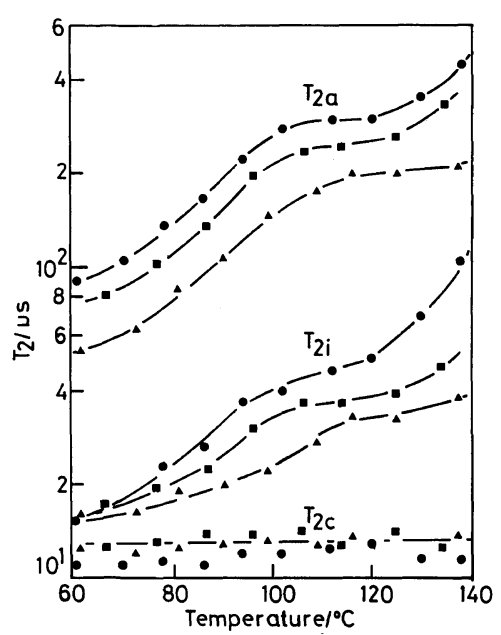

Figure 1. Temperature dependence of $T_{2 \mathrm{a}}, T_{2 \mathrm{i}}$, and $T_{2 \mathrm{c}}$ for the SGC series $(\mathrm{DR}=1(\mathbf{O}), 10(\square)$, and $17(\boldsymbol{\Delta}))$. 
scale. $T_{2 \mathrm{a}}$ changes markedly with temperature and draw ratio. All the samples exhibit an abrupt increase in $T_{2 \mathrm{a}}$ around $80^{\circ} \mathrm{C}$ which is probably due to a high frequency $(c a .10 \mathrm{kHz})$ manifestation of the glass-rubber transition of PP. ${ }^{13}$ At a given temperature, the $T_{2 \mathrm{a}}$ value decreases with increasing draw ratio. The intermediate $T_{2 \mathrm{i}}$ component also follows an exponential decay, and shows an abrupt increase around the temperature where $T_{2 \mathrm{a}}$ transition is observed. However, at a given draw ratio and temperature, the value of $T_{2 \mathrm{i}}$ is quite small compared with that of $T_{2 \mathrm{a}}$. These indicate that the $T_{2 \mathrm{i}}$ is responsible for the segmental motions in the fairly restricted noncrystalline regions. The shortest relaxation time $T_{2 \mathrm{c}}$ is associated with the crystalline regions, ${ }^{4}$ as suggested by the Gaussian form of the decay. The $T_{2 \mathrm{c}}$ shows no significant dependence on the temperature and draw ratio. The NMR measurements were also carried out for the MGC series. However, the data were quite similar to our previous NMR results of drawn polypropylene which was prepared from bulkcrystallized films by tensile drawing. ${ }^{4}$ Thus, the temperature dependence of $T_{2}$ for the MGC series was not presented in this paper. It seems that the difference of drawing technique has no appreciable effect on the chain mobility in the drawn PP. Around $110^{\circ} \mathrm{C}, T_{2 \mathrm{a}}$ and $T_{2 \mathrm{i}}$ as well as $T_{2 \mathrm{c}}$ for both series were weakly dependent on temperature. In order to evaluate the effect of drawing on the mobility of the noncrystalline segments, $T_{2 \mathrm{a}}$ and $T_{2 \mathrm{i}}$ at $110^{\circ} \mathrm{C}$ are plotted against draw ratio in Figure 2: In the MGC series, both $T_{2 \mathrm{a}}$ and $T_{2 \mathrm{i}}$ decrease sharply up to draw ratio of 10 , and then $T_{2 \mathrm{i}}$ becomes saturated, whereas $T_{2 \mathrm{a}}$ continues a decrease with further increase in the draw ratio. These results indicate that the structure of intermediate and amorphous phases change drastically with drawing up to draw ratio of 10 , above which the intermediate phase reaches a limiting structure. Both $T_{2 \mathrm{a}}$ and $T_{2 \mathrm{i}}$ of the SGC series also decrease with increasing draw ratio, however, the changes in $T_{2 \mathrm{i}}$ are rather small

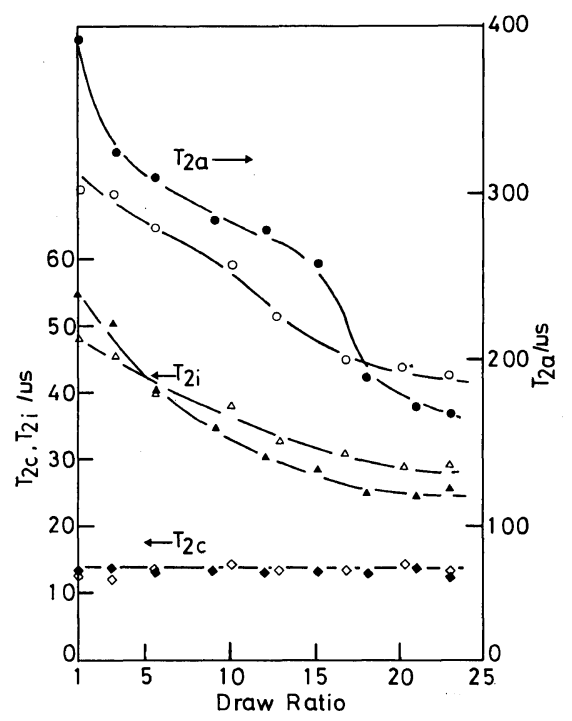

Figure 2. Draw ratio dependence of $T_{2 \mathrm{a}}, T_{2 \mathrm{i}}$, and $T_{2 \mathrm{c}}$ at $110^{\circ} \mathrm{C}$ for the MGC series $(\Delta \Delta)$ and the SGC series $(\bigcirc \triangle \diamond)$.

compared with those for MGC series. In addition, the values of $T_{2 \mathrm{i}}$ and $T_{2 \mathrm{a}}$ at higher draw ratio for SGC series are longer than those for MGC series. The results demonstrate that at higher draw ratio, the tautness of the noncrystalline molecules is higher for MGC than for SGC series.

The fraction of $T_{2 \mathrm{a}}, T_{2 \mathrm{i}}$ and $T_{2 \mathrm{c}}$ components $\left(F_{\mathrm{a}}, F_{\mathrm{i}}\right.$ and $\left.F_{\mathrm{c}}\right)$ also change with measurement temperature, draw ratio, and pre-drawn morphology. Below the glass-rubber transition temperature, $F_{\mathrm{c}}$ includes both crystalline and noncrystalline phases in which segmental mobility is almost frozen in. Between the glass rubber transition and crystalline relaxation temperature $F_{\mathrm{c}}$ corresponds to the massfraction crystallinity at the measurement temperature: In order to discuss the structural changes during drawing, each fraction for both series at $100^{\circ} \mathrm{C}$ is plotted against draw ratio in Figure 3. For the SGC series $F_{\text {a }}$ decreases gradually, and simultaneously $F_{\mathrm{i}}$ increases with increasing draw ratio. The results combined with $T_{2}$ data (Figure 2) indicate that with increasing draw ratio, the tautness of 


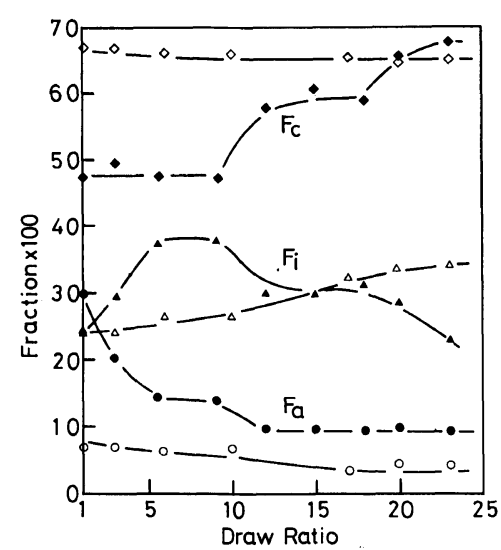

Figure 3. Draw ratio dependence of $F_{\mathrm{a}}, F_{\mathrm{i}}$, and $F_{\mathrm{c}}$ at $100^{\circ} \mathrm{C}$ for the MGC series $(\Delta \Delta)$ and the SGC series $(\bigcirc \triangle \diamond)$

amorphous and intermediate phases increases, and simultaneously a part of the amorphous phase is transformed into intermediate phase. The $F_{\mathrm{c}}$ remains almost constant for the draw ratio investigated. The lack of substantial changes in $F_{\mathrm{c}}$ with draw ratio suggests that the taut molecules in the SGC series are not in sufficiently good lateral register to form intercrystalline bridges of crystalline nature. For the MGC series, up to draw ratio of 9 , the increase in $F_{\mathrm{i}}$ is accompanied by a decrease in $F_{\mathrm{a}}$ with $F_{\mathrm{c}}$ remaining unchanged. At higher draw ratio, however, $F_{\mathrm{a}}$ is almost constant, and stepwise rises in $F_{\mathrm{c}}$ are accompanied by corresponding drop in $F_{\mathrm{i}}$. In this draw ratio range, the $T_{2 \mathrm{i}}$ associated with $F_{\mathrm{i}}$ is almost constant and the value is shorter than that for the SGC series (see Figure 2). Therefore, as draw ratio increases, the net effect is that some of the highly strained molecules are transformed into crystalline bridges.

\section{Temperature Dependence of Linear Thermal Expansion}

The changes in the noncrystalline structure during deformation were also investigated by the thermomechanical analysis. The temperature dependence of linear thermal expansivity along the drawing direction $\left(\beta_{\|}\right)$for the two

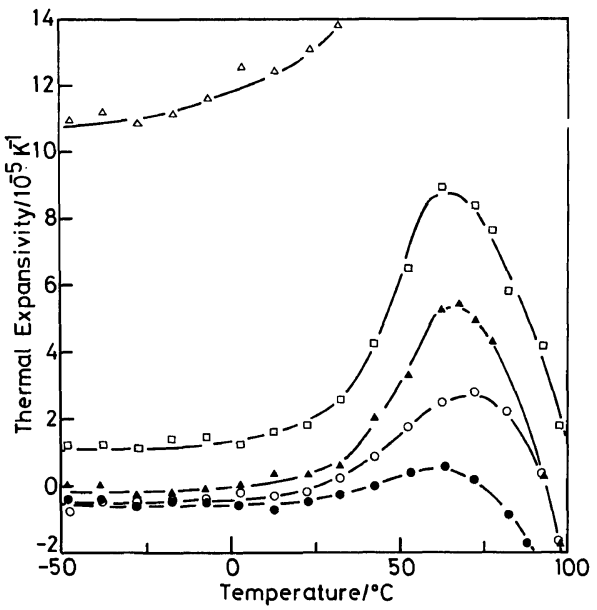

Figure 4. Temperature dependence of thermal expansivities along the drawing direction for the SGC series $(\mathrm{DR}=1(\triangle), 6(\square), 9(\Delta), 15(\bigcirc)$, and $23(\bigcirc))$.

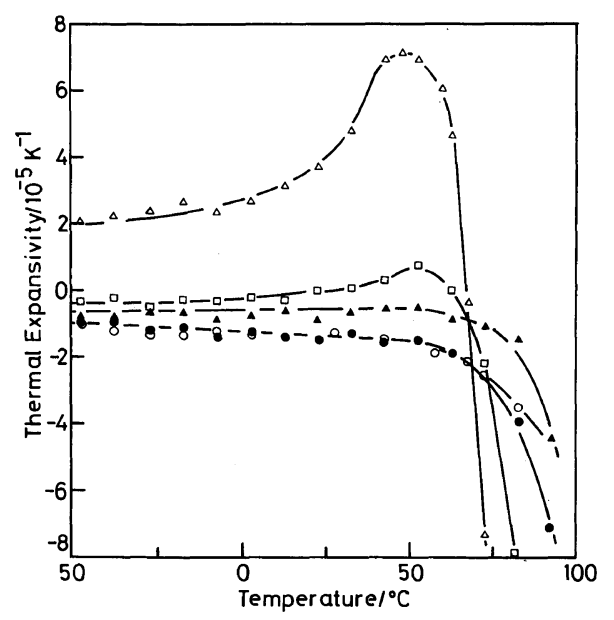

Figure 5. Temperature dependence of thermal expansivities along the drawing direction for the MGC series $(\mathrm{DR}=3(\triangle), 6(\square), 8(\Delta), 15(\bigcirc)$, and $23(\bigcirc))$.

series are shown in Figures 4 and 5. The $\beta$ for isotropic PP shows a sharp increase around $0^{\circ} \mathrm{C}$. This rise is associated with the onset of micro-Brownian motions of the noncrystalline segments. The $\beta_{\|}$for the samples with low draw ratio also shows a rapid increase which is followed by a drop at higher temperature resulting in a peak at $60-80^{\circ} \mathrm{C}$. At higher draw ratio, the sharp increase in $\beta_{\|}$becomes less prominent especially for the MGC series. In 
the highly drawn samples, the micro-Brownian motions of the noncrystalline segments are severely restricted, which suppress the large increase in $\beta_{\|}$, indicating that the tautness of the noncrystalline segments is higher for the MGC than for SGC series in agree with the NMR results. The large decrease in $\beta_{\|}$at higher temperature, already observed for a larger number of drawn semicrystalline polymers, ${ }^{14-16}$ has been attributed to two factors of development of crystalline bridges and the effects of entropic rubber-elastic contraction. ${ }^{17}$ Although the axial thermal expansivity of the crystals $\left(\beta_{\|}{ }^{\mathrm{c}}\right)$ of PP has not yet been determined, the value was suggested to be $\sim-1 \times 10^{-5}$ $\mathrm{K}^{-1} \cdot{ }^{18}$ In the present study, all the drawn samples showed a large drop of $\beta_{\|}$above $60-80^{\circ} \mathrm{C}$, further, the values above $100^{\circ} \mathrm{C}$ exceed the value of $\beta_{\|}{ }^{c}$. The results suggest that the large drop of $\beta_{\|}$is primarily arised from the rubber elastic contraction of the oriented noncrystalline segments. In the drawn PP, the existence of the taut molecules or crystalline bridges has been suggested. ${ }^{7,11}$ Our present NMR results for the MGC series also suggested the transformation of highly strained noncrystalline molecules into crystalline bridges at higher deformation ratio. The large rubber elastic effect observed in this study implies that the axial length of the bridges is not enough and/or the bridge content is not sufficiently large to regist the contraction.

Below the glass transition temperature the rubber elastic effect is absent and the $\beta_{\|}$is directly related to the chain orientation. Figure 6 shows the draw ratio dependence of $\beta_{\|}$at $-40^{\circ} \mathrm{C}$ for both series. It is seen that the $\beta_{\|}$ shows a sharp decrease up to draw ratio of 10 . The limiting value of $\beta_{\|}$for the MGC series is $1 \times 10^{-5} \mathrm{~K}^{-1}$, which is lower than that for the SGC series. Unfortunately, detailed analysis of the observed $\beta_{\|}$is not possible since relevant data such as axial thermal expansivities of the crystalline and noncrystalline phases are not available, but it is certain that the changes in the $\beta_{\|}$with draw ratio arise from the chain

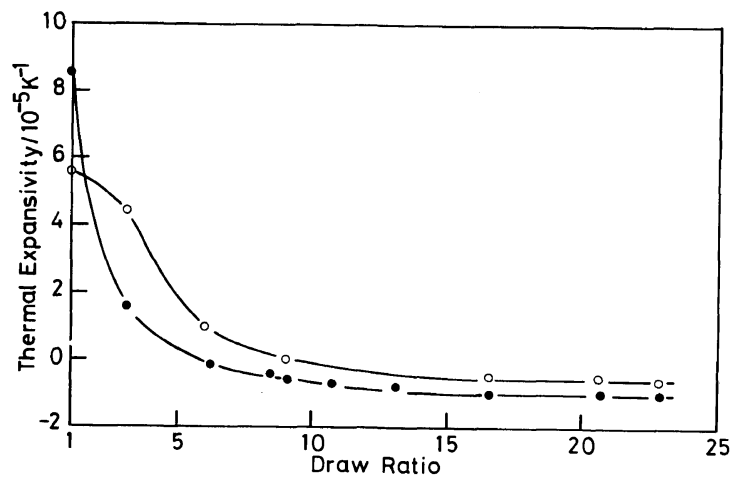

Figure 6. Draw ratio dependence of the thermal expansivities along the drawing direction at $-40^{\circ} \mathrm{C}$ for the MGC series (O) and the SGC series (O).

alignment in the crystalline and noncrystalline phases and from the production of intercrystalline bridges. Thus, the present data indicate that the average chain orientation is higher for MGC than for SGC series.

\section{Mechanical Properties}

The tensile modulus for the MGC and SGC series is plotted against draw ratio in Figure 7. For both series, the modulus increases with increasing draw ratio. At any draw ratio, the modulus for the MGC is larger than for SGC series. This trend becomes more prominent at higher draw ratio. The crystalline orientation function $\left(f_{\mathrm{c}}\right)$ evaluated from X-ray for both series is plotted against draw ratio in Figure 8. At low draw ratio $(<12)$, the $f_{\mathrm{c}}$ for both series increases rapidly with increasing draw ratio. At higher draw ratio $(>12)$ of both series, the $f_{\mathrm{c}}$ becomes saturated. At any draw ratio, the $f_{\mathrm{c}}$ for MGC is higher than for SGC series, however, the difference is not so pronounced for higher draw ratio. It is generally accepted that the tensile modulus along the drawing direction is a function of chain alignment in both crystalline and noncrystalline regions and the number of taut tie molecules or crystalline bridges. The fact that the continuous increase in the modulus at higher draw ratio where the $f_{\mathrm{c}}$ is almost saturated, is considered to be closely related to the structure of the non- 


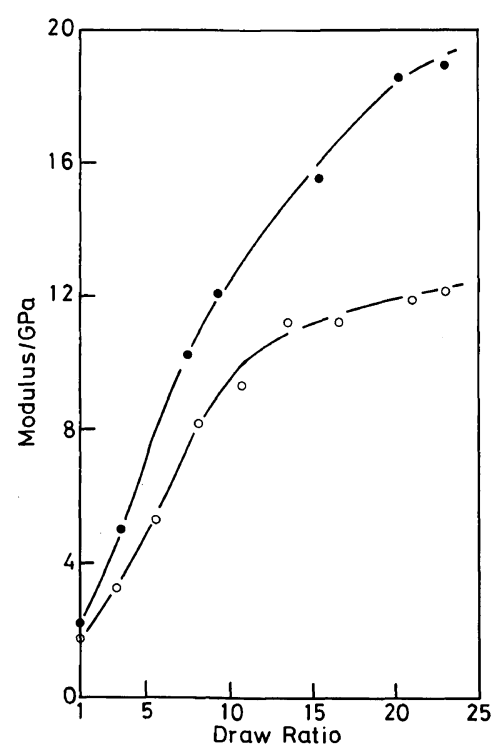

Figure 7. Draw ratio dependence of the tensile modulus at room temperature for the MGC series (O) and the SGC series $(O)$.

crystalline regions in the drawn materials. As revealed by NMR data, the molecular constraint on the highly strained noncrystalline component ( $T_{2 \mathrm{i}}$ component) in the MGC series approaches limit at higher draw ratio, and simultaneously a part of this phase is incorporated into crystalline regions, suggesting a crystalline bridge formation. However, in the SGC series such changes were less clear. As shown in Figure 6 and 8, averaged chain orientation and crystalline orientation functions are higher for the MGC than for SGC series, which may produce the difference in the modulus at lower draw ratio. However, at a higher draw ratio, the difference may be arised from both the difference in the fraction of crystalline bridges and the tautness of the highly constrained noncrystalline molecules ( $T_{2 \mathrm{i}}$ component).

All the data obtained clearly showed that the initial crystalline morphology has a remarkable effect on the structure and properties of drawn PP. At low draw ratio, the deformation mechanism of spherulitic materials is

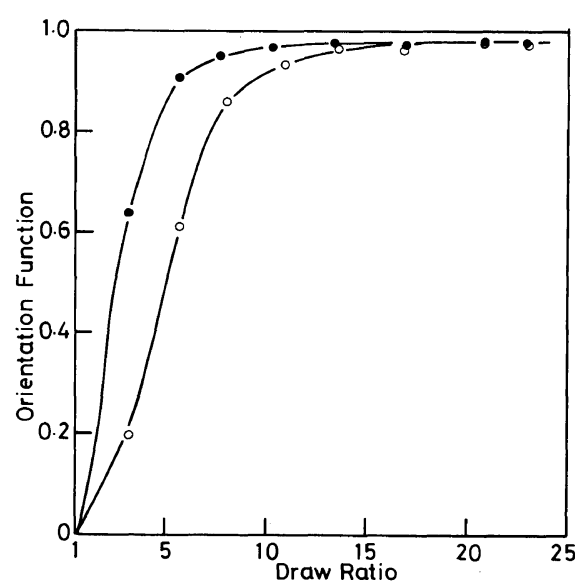

Figure 8. Draw ratio dependence of the crystalline orientation function for the MGC series (O) and the SGC series (O).

more complex than that of solution grown crystals. Thus, it is difficult to explain the high efficiency of draw observed in the MGC series. The proposed deformation mechanism at middle to high draw ratio is the fibril formation by chain slip and chain unfolding followed by interfibrillar slip. ${ }^{1,2}$ It is widely accepted that the number of intra and interlamellar tie molecules is larger in bulk-crystallized spherulitic materials than in solution grown crystals. Such tie molecules act as an axial force transmitter, which may facilitate the fibril formation by chain slip and chain unfolding. At this stage, the number of newly formed taut tie molecules increases, which may produce the high efficiency of draw.

Kanamoto et al. ${ }^{19}$ studied the effect of initial morphology on the crystalline state extrusion of high density polyethylene and reported that the maximum attainable draw ratio was higher for solution grown crystals than for spherulitic materials. They suggested that interfacial friction adhesion between crystalline lamellae is a primary mechanism for drawing. As described in the experimental section, SGC series could be easily drawn up to draw ratio of 23 , however, the attainable maximum draw ratio of MGC by the solid 
state co-extrusion method was 16 . The results indicate that the deformability is higher for SGC than for MGC in analogy with linear polyethylene.

\section{REFERENCES}

1. A. Peterlin, J. Mater. Sci., 6, 490 (1971).

2. A. Peterlin, Polym. Eng. Sci., 19, 118 (1979).

3. M. Ito, T. Kanamoto, K. Tanaka, and R. S. Porter, Macromolecules, 14, 1779 (1981).

4. M. Ito, H. Serizawa, K. Tanaka, W. P. Leung, and C. L. Choy, J. Polym. Sci., Polym. Phys. Ed., 21, 2299 (1983).

5. D. L. M. Cransfield, G. Capaccio, and I. M. Ward, Polym. Eng. Sci., 16, 721 (1975).

6. W. N. Taylor and E. S. Clark, Polym. Eng. Sci., 18, 518 (1978).

7. M. Kamezawa, K. Yamada, and M. Takayanagi, $J$. Appl. Polym. Sci., 24, 1227 (1979).

8. K. Yamada, M. Kamezawa, and M. Takayanagi, $J$. Appl. Polym. Sci., 26, 49 (1981).

9. C. L. Choy, F. C. Chen, and K. Young, J. Polym.
Sci., Palym. Phys. Ed., 19, 335 (1981).

10. W. P. Leung, C. C. Chan, F. C. Chen, and C. L. Choy, Polymer, 21, 1148 (1980).

11. W. P. Leung and C. L. Choy, J. Polym. Sci., Polym. Phys. Ed., 21, 725 (1983).

12. P. D. Griswold, A. E. Zachariades, and R. S. Porter, presented at the Stress Induced Crystallization Symposium, Midland Macromolecular Institute, M. I., 1977.

13. R. G. Saba, J. A. Sauer, and A. E. Woodward, $J$. Polym. Sci., A, 1, 1483 (1963).

14. R. Meredith and B. S. Hsu, J. Polym. Sci., 61, 271 (1962).

15. Y. Ohzawa and Y. Wada, Rep. Prog. Polym. Phys. Jpn., 7, 193 (1964).

16. B. H. Kim and R. De Betist, J. Polym. Sci., Polym. Lett. Ed., 11, 121 (1973).

17. C. L. Choy, F. C. Chen, and K. Young, J. Polym. Sci., Polym. Phys. Ed., 19, 335 (1981).

18. I. M. Ward, "Developments in Oriented Polymers I," Applied Science Publishers, Essex, U. K. 1982, p 135.

19. T. Kanamoto, E.S. Sherman, and R. S. Porter, Polym. J., 11, 497 (1979). 